\title{
EDITORIAL AND COMMENT Hepatitis C Virus Cure and Obesity: Watch the Weight
}

\author{
Nizar A. Mukhtar, MD ${ }^{7}$ and Rena K. Fox, $M D^{2}$ \\ 'Department of Gastroenterology, Kaiser Permanente San Francisco Medical CenterSan Francisco, CA, USA; ${ }^{2}$ Division of General Internal \\ Medicine, Department of Medicine, University of California San Francisco School of Medicine San Francisco, CA, USA.
}

J Gen Intern Med 35(10):2836-7

DOI: $10.1007 / \mathrm{s} 11606-020-06004-9$

(c) Society of General Internal Medicine 2020

$\mathrm{O}$ besity has reached an astounding prevalence in the general population, and the burden of disease worsens with increasing age. Through unclear mechanisms, greater body mass index (BMI) is associated with an increased rate of death from all causes even when accounting for confounders such as smoking history or presence of comorbid illness. ${ }^{1}$ Obesity is the primary risk factor for the development of non-alcoholic fatty liver disease (NAFLD), which is becoming the most common cause of chronic liver disease and hepatocellular carcinoma (HCC) as well as the leading indication for liver transplantation in the U.S. Development of concomitant NAFLD is associated with more rapid progression of hepatic fibrosis in patients with other causes of liver disease, including chronic viral hepatitis and alcoholic liver disease.

It is therefore important to take note of the current prospective observational cohort study by Do, et al. ${ }^{2}$ of an impressively large sample of over 11,000 veterans with chronic hepatitis $\mathrm{C}$ virus (HCV) infection successfully treated with direct acting antiviral (DAA) medications, showing that more than $20 \%$ of patients gained $10 \mathrm{lbs}$ or more within two years of achieving a sustained virologic response (SVR). The prevalence of obesity in this HCV-infected cohort was $36.8 \%$ at baseline, significantly lower than the $41 \%$ prevalence of obesity among the overall veteran population. Weight gain was more commonly observed with younger age, baseline overweight or obesity, cirrhosis, and moderate alcohol use.

Several questions arise from this study. First, is the excess weight gain specifically related to the use of DAA therapy? Many classes of medications are associated with weight gain, such as antipsychotics, antidepressants, hypoglycemic agents, glucocorticoids, and beta blockers, and typically, the weight gain can be reversed after discontinuation of treatment. Some HIV antiretrovirals are associated with weight gain, but as a class, antiviral drug-induced weight gain is not a common finding. The authors attempted to remove the DAA itself from the equation by determining baseline weight at three months after DAA treatment was completed, rather than before DAA treatment, and then to determine the change in weight with the measurement again two

Published online July 6, 2020 years later. However, in order to determine if the weight gain effect is specifically due to the use of DAA therapy, the patients should have been compared to historical controls of HCV patients who were untreated and HCV patients achieving SVR using interferon-based regimens.

Second, is the excess weight gain due to the eradication of HCV? The authors postulated on potential mechanisms by which an HCV cure could lead to weight gain, including removal of chronic inflammation or infection, increased nutritional intake, and/or improved hepatic anabolic function with associated increase in skeletal muscle mass. Along these lines, the hypermetabolic state and protein catabolism that lead to sarcopenia and weight loss in patients with end-stage liver disease often reverse following liver transplantation such that nearly a third of patients become obese three years following surgery. ${ }^{3}$ Similarly, the molecular and metabolic derangements giving way to sarcopenia in patients with alcoholic cirrhosis can resolve with abstinence from alcohol and improved nutritional status, such that patients regain muscle mass. ${ }^{4}$ That said, if eradication of chronic $\mathrm{HCV}$ produces these effects, then we might expect to see similar effects with successful suppression of chronic HBV infection. However, there are no reports in the literature to date suggesting an association between HBV antiviral therapy and excess weight gain. Nevertheless, it is plausible that HCV-specific factors that shape a unique metabolic profile are eliminated following cure, leading to weight gain.

Third, is the excess weight gain due to increases in fat stores or muscle mass? It is well-established that chronic liver disease is associated with a host of nutritional abnormalities and body composition changes that vary as a function of sex and severity of liver disease. Indeed, up to a third of a large cohort of patients with cirrhosis were found to have signs of nutritional depletion with significant reduction in fat and/or muscle stores. ${ }^{5}$ Men with cirrhosis experience more dramatic reductions in muscle mass than women, and sarcopenia has been shown to be associated with poor outcomes, including higher waitlist and post-transplant mortality. While increased visceral adiposity may impose deleterious consequences, including the development of non-alcoholic steatohepatitis, increased subcutaneous adipose tissue may offer protection from metabolic derangements such as insulin resistance. Indeed, in a recent study of nearly 700 patients with cirrhosis, decreased subcutaneous adipose tissue was associated with higher mortality among women, ${ }^{6}$ and similar findings have been described in 


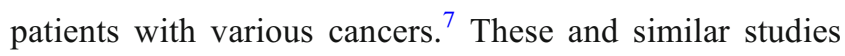
highlight the complex and significant relationship between body composition and health outcomes, and without the observed changes in muscle mass and distribution of fat, it is difficult to speculate on the clinical implications of the weight gain observed in patients with HCV treated with DAA therapy in this study.

Fourth, do HCV patients who achieve SVR have a change in health-related behaviors that contribute to weight gain? Patients treated with DAAs report increased health-related quality of life ${ }^{8}$ and increased motivation to pursue tobacco cessation, control of hypertension, and improvement in other chronic medical conditions. The prevalence of smoking is strikingly high at $67 \%$ in $\mathrm{HCV}$-infected veterans, ${ }^{9}$ and smoking cessation is associated with increased caloric intake and weight gain. As such, it would be interesting to know if health behaviors such as tobacco use changed in this cohort following $\mathrm{HCV}$ cure, potentially being one of the factors contributing to a change in eating behavior.

Finally, could the weight gain observed in this 2-year time period be due to the aging of this cohort and not be related to their HCV or DAA exposure? The long-term risk of becoming obese over one's life is high. In a prospective cohort study of 4117 normal-weight men and women age 30-59 years, 5-9\% of both groups became obese within 4 years. ${ }^{10}$ In the current study evaluating a population of largely middle-aged men with no comparison group, it is possible the observed weight gain represents the natural history of this group and is unrelated to their HCV status. Moreover, since the cohort in the current study had a lower prevalence of obesity than the overall veteran population, perhaps with the eradication of $\mathrm{HCV}$, there is a "catch up" effect on weight, whereby the weight gain corrects for weight loss incurred prior to treatment. A control group of patients without $\mathrm{HCV}$ infection and matched for initial age and BMI could have been used to help determine if this was the natural course of weight gain over this time period. Additionally, a control group would help to determine if the groups were at similar weights over a longer period, regardless of $\mathrm{HCV}$ status.

The lack of a control group detracts from the study findings, but the authors provided a rationale for not including one. A control group that was $\mathrm{HCV}$-infected but not treated with DAAs would lead to concerns that the untreated cohort had medical or social problems that made them poor candidates for DAA therapy. Similarly, a control group that was HCVinfected and treated with DAAs but did not achieve SVR would lead to concerns that the non-SVR group had behavioral problems that led to treatment failure.

This study highlights an important association of $\mathrm{HCV}$ cure following DAA therapy with weight gain that warrants further exploration. It is clear that achieving SVR in patients with chronic HCV results in reduced rates of cirrhosis, hepatic decompensation, HCC, and liver-related mortality, and if excess weight gain is truly an unintended consequence of curing $\mathrm{HCV}$, it is imperative to ascertain if this weight gain is necessarily causing adverse health outcomes. The authors are clearly not advocating that DAA treatment be withheld for concern of weight gain, yet providing DAA treatment may mean we need to have a heightened awareness of the risks of obesity and therefore NAFLD and associated cardiovascular morbidity. The continually growing obesity epidemic makes it one of the essential responsibilities of physicians to consider all shortand long-term side effects or outcomes of medications and to balance the benefits of treatment with the potential for weight gain and to help support patients in efforts to avoid excess weight gain, throughout adulthood.

Corresponding Author: Rena K. Fox, MD; Division of General Internal Medicine, Department of Medicine, University of California San Francisco School of Medicine San Francisco, CA, USA (e-mail: rena.fox@ucsf.edu).

\section{Compliance with Ethical Standards:}

Conflict of Interest: The authors report no affiliation or involvement in potential conflicts of interest.

\section{REFERENCES}

1. Berrington de Gonzalez A, Hartge $\mathbf{P}$, Cerhan JR, et al. Body-mass index and mortality among 1.46 million white adults [published correction appears in N Engl J Med. 2011 Sep 1;365(9):869]. N Engl J Med. 2010;363(23):2211-2219.

2. Do A, Esserman DA, Krishnan S, et al. Excess weight gain after cure of hepatitis $\mathrm{C}$ infection with direct-acting antivirals. J Gen Intern Med. 2020; doi:https://doi.org/10.1007/s11606-020-05782-6.

3. Richards J, Gunson B, Johnson J, Neuberger J. Weight gain and obesity after liver transplantation. Transpl Int. 2005; 18(4):461-6.

4. Dasarathy S. Nutrition and alcoholic liver disease: Effects of alcoholism on nutrition, effects of nutrition on alcoholic liver disease, and nutritional therapies for alcoholic liver disease. Clin Liver Dis. 2016;20(3):535-550.

5. Nutritional status in cirrhosis. Italian Multicentre Cooperative Project on Nutrition in Liver Cirrhosis. J Hepatol. 1994;21(3):317-25.

6. Ebadi M, Tandon P, Moctezuma-Velazquez C, et al. Low subcutaneous adiposity associates with higher mortality in female patients with cirrhosis. J Hepatol. 2018;69(3):608-616.

7. Ebadi M, Martin L, Ghosh S, et al. Subcutaneous adiposity is an independent predictor of mortality in cancer patients. $\mathrm{Br} \mathrm{J}$ Cancer. 2017;117(1): 148-155.

8. Juanbeltz R, Martínez-Baz I, San Miguel R, Goñi-Esarte S, Cabasés JM, Castilla J. PLoS One. 2018;13(10):e0205277

9. Chew KW, Bhattacharya D, McGinnis KA, et al. Short communication: coronary heart disease risk by Framingham risk score in hepatitis $\mathrm{C}$ and HIV/hepatitis C-coinfected persons. AIDS Res Hum Retrovir. 2015;31(7):718-722.

10. Vasan RS, Pencina MJ, Cobain M, Freiberg MS, D'Agostino RB. Estimated risks for developing obesity in the Framingham Heart Study. Ann Intern Med. 2005; 143(7):473-80

Publisher's Note: Springer Nature remains neutral with regard to jurisdictional claims in published maps and institutional affiliations. 